\title{
AN EXAMPLE FOR HOMOTOPY GROUPS WITH COEFFICIENTS
}

\section{MARTIN ARKOWITZ}

In this note we present two spaces $X$ and $Y$ all of whose homotopy groups are isomorphic, but whose homotopy groups with coefficients are not isomorphic for a certain coefficient group. ${ }^{1}$ This example depends on the fact that the universal coefficient sequence [2], which relates the ordinary homotopy groups to those with coefficients, does not split. The spaces $X$ and $Y$ will be 1-connected, CW-complexes and the coefficient group will be $Z_{m}$, the integers modulo $m$.

We adopt the following notation: $M(G, p)$ denotes a Moore complex of type $(G, p)$ (i.e., a space with a single nonvanishing homology group $G$ in dimension $p$ ) and $K(G, p)$ denotes an Eilenberg-MacLane complex of type $(G, p)$ (i.e., a space with a single nonvanishing homotopy group $G$ in dimension $p$ ). Recall that $\pi_{r}(G ; A)$, the $r$ th homotopy group of the space $A$ with coefficients in the group $G$, is the group of homotopy classes of base point preserving maps from $M(G, r)$ into $A$. If $Z$ is the group of integers, then $\pi_{r}(Z ; A)=\pi_{r}(A)$, the $r$ th homotopy group of $A$. Finally we recall the universal coefficient theorem [2] which asserts the exactness of the following sequence

(1) $0 \rightarrow \operatorname{Ext}\left(G, \pi_{r+1}(A)\right) \rightarrow \pi_{r}(G ; A) \rightarrow \operatorname{Hom}\left(G, \pi_{r}(A)\right) \rightarrow 0$.

Now let $\bar{X}=M\left(Z_{n}, r\right)$ and let $d$ be the greatest common divisor of $m$ and $n$. We shall always assume that $d$ is even, that 8 does not divide $m n$, and that $r$ is an integer $>2$. Under these conditions Barratt [1] has shown that $\pi_{r}\left(Z_{m} ; \bar{X}\right) \approx Z_{2 d}$. In addition, it is well known that $\pi_{r+1}(\bar{X}) \approx Z_{n} \otimes Z_{2}=Z_{2}$. Now let $X$ be the space obtained from $\bar{X}$ by attaching cells to kill all homotopy groups in dimensions $\geqq r+2$. Thus

$$
\begin{aligned}
& \pi_{i}(X)=0 \text { for all } i \leqq r-1 \text { and } \geqq r+2, \\
& \pi_{r}(X)=Z_{n} \text { and } \pi_{r+1}(X)=Z_{2} .
\end{aligned}
$$

Furthermore, since $M\left(Z_{m}, r\right)$ is an $(r+1)$-dimensional CW-complex and the $(r+2)$-skeleton of $X$ is $\bar{X}$, it follows from standard cellular approximation arguments that $\pi_{r}\left(Z_{m} ; X\right) \approx \pi_{r}\left(Z_{m} ; \bar{X}\right)$. Hence we have

$$
\pi_{r}\left(Z_{m} ; X\right) \approx Z_{2 d} \text {. }
$$

Next let $Y$ be a product of Eilenberg-MacLane spaces, $Y=K\left(Z_{n}, r\right)$

Received by the editors July 26, 1962 and, in revised form, November 14, 1962.

1 We have been informed that such an example was known to Eckmann and Hilton. 
$\times K\left(Z_{2}, r+1\right)$. Since $\pi_{i}(Y) \approx \pi_{i}\left(K\left(Z_{n}, r\right)\right) \oplus \pi_{i}\left(K\left(Z_{2}, r+1\right)\right)$, by (2) we have that $\pi_{i}(X)$ and $\pi_{i}(Y)$ are isomorphic for all $i$. Now we consider $\pi_{r}\left(Z_{m} ; Y\right)$. Clearly $\pi_{r}\left(Z_{m} ; Y\right) \approx \pi_{r}\left(Z_{m} ; K\left(Z_{n}, r\right)\right) \oplus \pi_{r}\left(Z_{m} ; K\left(Z_{2}, r+1\right)\right)$. By $(1), \pi_{r}\left(Z_{m} ; K\left(Z_{n}, r\right)\right) \approx \operatorname{Hom}\left(Z_{m}, Z_{n}\right)=Z_{d}$. Also by (1),

$$
\pi_{r}\left(Z_{m} ; K\left(Z_{2}, r+1\right)\right) \approx \operatorname{Ext}\left(Z_{m}, Z_{2}\right)=Z_{2} .
$$

Thus

$$
\pi_{r}\left(Z_{m} ; Y\right) \approx Z_{d} \oplus Z_{2} .
$$

A comparison of (3) and (4) shows that $\pi_{r}\left(Z_{m} ; X\right)$ is not isomorphic to $\pi_{r}\left(Z_{m} ; Y\right)$. But we have already seen that $\pi_{i}(X) \approx \pi_{i}(Y)$ for all $i$. This completes the example.

We observe that the spaces $X$ and $Y$ may be distinguished by invariants other than the $r$ th homotopy group with coefficients in $Z_{m}$. For instance, it is easily seen that $H_{r+1}(X)=0$ and $H_{r+1}(Y)=Z_{2}$.

In closing we note that the spaces $X$ and $Y$ serve as an example for homotopy groups with coefficients as defined by Katuta [3]. This is so since, for a finite coefficient group, Katuta's groups are the same as the ones we consider, except for a dimensional shift of one unit.

\section{REFERENCES}

1. M. G. Barratt, Track groups. II, Proc. London Math. Soc. (3) 5 (1955), 285329.

2. B. Eckmann and P. J. Hilton, Groupes d'homotopie et dualite. Coefficients, C. R. Acad. Sci. Paris 246 (1958), 2991-2993.

3. Y. Katuta, Homotopy groups with coefficients, Sci. Rep. Tokyo Kyoiku Daigaku Sect. A 7 (1960), 5-24.

Princeton University 